$r \geqq \sum_{p} r(p)$ then $G$ is quotient divisible.

Proof. For each $p, G / p G$ is a direct sum of cyclic groups of order $p: G / p G=\sum_{(i, p) \in I(p)} Z(x(i, p)+p G)$ for some elements $x(i, p) \in G$. Evidently, $r(p)=|I(p)|$. Since rank $p G=\operatorname{rank} G=r$ for each $p$, we may apply Theorem 2.1 with $N=\{p \mid p$ is a prime $\}, A_{p}=p G$ for each $p \in N$ and $B_{p}=G$ for each $p \in N$, and obtain a free group $F \subseteq G$ such that $F+p G=G$ for each $p$. Applying Lemma 3.3, with $S$ any basis of $F$, we conclude that $G$ is quotient divisible.

COROLlary 3.5. Any torsion free group of infinite rank is quotient divisible.

PROOF. $\sum_{p} r(p) \leqq \sum_{p} r=\aleph_{0} r=r$.

\title{
BIBLIOGRAPHY
}

1. R. A. Beaumont and R. S. Pierce, Torsion free rings, Illinois J. Math. 5 (1961), 61-98.

SYRACUSE UNIVERSITY

\section{CONVOLUTIONS OF SLOWLY OSCILLATING FUNCTIONS}

\section{J. P. TULL}

1. Introduction. In the study of asymptotic formulae for arithmetic functions we invariably come upon such functions as $x^{\alpha} \log ^{\beta} x$, li $x$, $x^{\alpha}(\log x)^{\beta}(\log \log x)^{\gamma}, x^{\alpha} \exp \left(\beta \log ^{\gamma_{0}} x\right)$. If $\alpha$ is complex and $\beta$ and $\gamma$ are real numbers $\left(\gamma_{0}<1\right)$ then these functions are of the form $x^{\alpha} L(x)$, where $L$ is a slowly oscillating function; i.e., a continuous positive valued function on $\left[x_{0}, \infty\right)$ for some $x_{0} \geqq 1$ such that

$$
\lim _{x \rightarrow \infty} L(c x) / L(x)=1
$$

for each $c>0$. A common approach to asymptotic formulae is the convolution method of Landau which was formulated into a general theorem by the author [4]. The resulting main terms involve convolutions of functions $x^{\alpha} L(x)$. In the present paper we shall give conditions under which such convolutions are also in the form $x^{\alpha} L(x)$ or nearly so.

Now it is known $[1 ; 3]$ (see [2] for other properties) that a function $L$ on $\left[x_{0}, \infty\right)$ is slowly oscillating if and only if there exist continuous functions $\rho$ and $\delta$ on $\left[x_{0}, \infty\right)$ such that $\rho(x)>0, \rho(x) \rightarrow \rho_{0}>0$, $\delta(x) \rightarrow 0$ as $x \rightarrow \infty$ and

Received by the editors February 24, 1961. 


$$
L(x)=\rho(x) \exp \int_{x_{0}}^{x} t^{-1} \delta(t) d t .
$$

We shall be concerned in particular with the special slowly oscillating functions having the form

$$
L(x)=\rho \exp \int_{1}^{x} t^{-1} \delta(t) d t
$$

where $\rho>0$ is constant and $\delta$ is a bounded measurable function with $\delta(x)=o(1)$ as $x \rightarrow \infty$. By a convolution of two functions $A$ and $B$ we shall mean one of

$$
\int_{a}^{x / a} A(x / u) d B(u) \text { or } \int_{a}^{x / a} k(u) A(x / u) B(u) d u .
$$

The former of these expressions is called the Stieltjes resultant of $A$ by $B$ on $\left[a^{2}, \infty\right)$.

2. A convolution theorem. Suppose $\alpha$ and $\beta$ are real numbers with $\beta \neq 0$ and that $L$ and $M$ are slowly oscillating with $M$ special. Then for sufficiently large $a \geqq 1$ the Stieltjes resultant on $\left[a^{2}, \infty\right)$ of $x^{\alpha} L(x)$ by $x^{\beta} M(x)$ has the form $\beta x^{\gamma} N(x)$ where $\gamma=\max (\alpha, \beta)$ and $N(x)$ is slowly oscillating for $x>a^{2}$. Further, $N(x)$ is asymptotically proportional to $L(x)$ if $\alpha>\beta$ and to $M(x)$ if $\alpha<\beta$.

Proof. If $M$ is special then we have

$$
M(x)=\rho \exp \int_{1}^{x} t^{-1} \delta(t) d t
$$

with $\rho>0, \delta(t)=o(1)$ as $t \rightarrow \infty$. Choose $a \geqq 1$ so that $|\delta(t)|<|\beta|$ for all $t \geqq a$ and thus $\beta+\delta(t)$ remains constant in sign for $t \geqq a$. Now the Stieltjes resultant in question is

$$
\begin{aligned}
\int_{a}^{x / a} & (x / u)^{\alpha} L(x / u) d\left(u^{\beta} M(u)\right) \\
& =\int_{a}^{x / a}(x / u)^{\alpha} L(x / u) u^{\beta-1} M(u)(\beta+\delta(u)) d u \\
& =x^{\alpha} \int_{a}^{x / a} u^{-(\alpha-\beta)-1} L(x / u) M(u)(\beta+\delta(u)) d u \\
& =x^{\beta} \int_{a}^{x / a} u^{-(\beta-\alpha)-1} M(x / u)(\beta+\delta(x / u)) L(u) d u .
\end{aligned}
$$


If $\alpha>\beta$ we apply Lemma 1 below to (1) with

$$
A(x)=x^{-(\alpha-\beta)-1} M(x)(\beta+\delta(x))=O\left(x^{-(\alpha-\beta)-1+\varepsilon}\right)
$$

for each $\epsilon>0$ (e.g., $\epsilon=(\alpha-\beta) / 2$ ). Thus the resultant is asymptotic to

$$
x^{\alpha} L(x) \int_{a}^{\infty} u^{-(\alpha-\beta)-1} M(u)(\beta+\delta(u)) d u
$$

as $x \rightarrow \infty$, if $\alpha>\beta$. (The integral is different from 0 by our choice of $a$.) Similarly, if $\beta>\alpha$, we apply Lemma 1 to (2) and find the resultant asymptotic to

$$
\beta x^{\beta} M(x) \int_{a}^{\infty} u^{-(\beta-\alpha)-1} L(u) d u .
$$

If $\alpha=\beta$, (1) gives

$$
x^{\alpha} \int_{a}^{x / a} u^{-1} L(x / u) M(u)(\beta+\delta(u)) d u,
$$

to which we apply Lemma 2.

3. Two lemmas. Lemma 1. Suppose $A$ and $B$ are measurable functions on $[a, \infty)(a>0)$ with

$$
A(x)=O\left(x^{-\kappa}\right)
$$

for some $\kappa>1$. Suppose $B$ is positive valued, bounded on each bounded interval and asymptotic to a slowly oscillating function. Then

$$
\lim _{x \rightarrow \infty} \int_{a}^{x / a} A(u)(B(x / u) / B(x)) d u=\int_{a}^{\infty} A(u) d u .
$$

Proof. Set $B(x)=0$ for $0 \leqq x<a$ and apply the Lebesgue dominated convergence theorem to

$$
\int_{a}^{x / a} A(u)(B(x / u) / B(x)) d u=\int_{a}^{\infty} A(u)(B(x / u) / B(x)) d u .
$$

Now for all $u \geqq a$

$$
\lim _{x \rightarrow \infty} A(u) B(x / u) / B(x)=A(u) .
$$

With the aid of (1.2), for each $\epsilon>0$

$$
B(x / u) / B(x)=O\left(u^{e}\right)
$$

uniformly for $u \geqq a, x \geqq K$ (sufficiently large), and so we have 


$$
A(u) B(x / u) / B(x)=O\left(u^{-(x-e)}\right)
$$

with $0<\epsilon<\kappa-1$. Thus since $u^{-(x-e)}$ is absolutely integrable over $[a, \infty),(2)$ and (3) imply (1).

Lemma 2. Suppose $L$ is slowly oscillating and $B$ is measurable and asymptotic to a slowly oscillating function. Then for sufficiently large a

$$
N(x)=\int_{a}^{x / a} u^{-1} L(x / u) B(u) d u=\int_{a}^{x / a} u^{-1} L(u) B(x / u) d u
$$

is slowly oscillating for $x \geqq x_{0}>a^{2}$.

The proof is elementary. We use the fact that $\int_{a}^{x} u^{-1} L(u) d u$ is slowly oscillating and that

$$
\lim _{x \rightarrow \infty} B(c x) / B(x)=1
$$

uniformly for $k_{1} \leqq c \leqq k_{2}$ for each $k_{2}>k_{1}>0$.

4. Remarks. If $\alpha=\beta=0$ we have the Stieltjes resultant of $L$ by $M$. We can show it slowly oscillating under various hypotheses. In particular if $L$ and $M$ are both special and nondecreasing ( $M$ nonconstant).

Note that $A$ in Lemma 1 need not be real-valued. Thus if $\alpha$ and $\beta$ are complex with $R \alpha \neq \Re \beta$ the Stieltjes resultant of $x^{\alpha} L(x)$ by $x^{\beta} M(x)$ is $x^{\gamma} N(x)$ with $N(x)$ complex-valued but asymptotically proportional to $L(x)$ or $M(x)$ according as $R \alpha>\Re \beta$ or $\Re \beta>\Re \alpha$. $\gamma$ would be $\alpha$ in the former case and $\beta$ in the latter.

\section{BiBLIOGRAPHY}

1. J. Karamata, Sur une mode de croissance regulière des fonctions, Mathematica (Cluj) 4 (1930), 38-53.

2. E. E. Kohlbecker, Weak asymptotic properties of partitions, Trans. Amer. Math. Soc. 88 (1958), 346-365.

3. J. Korevaar, T. van Aardenne-Ehrenfest, and N. G. de Bruijn, A note on slowly oscillating functions, Nieuw Arch. Wiskunde 23 (1949), 77-86.

4. J. P. Tull, Dirichlet multiplication in lattice-point problems. II. Pacific J. Math. 9, no. 2 (1959), 609-615.

Oho State University 